economic decline. Without economic growth there would be no profits, hence no taxes, no public services, no pensions or state benefits - and no research grants! John Wright

Department of Earth Sciences, Open University, Milton Keynes MK7 6AA, UK

\section{DNA committee is model for bioterrorism debate}

Sir - There is much debate about how to protect the public from bioterrorism while maintaining the open exchange of biomedical research findings (see, for example, Nature 419, 99 and 769; 2002). There have been several calls by scientists and science journalists in the media for a 'summit conference' to discuss these concerns and to establish a US national forum to guide federal policy on scientific research with possible bioterrorism applications.

Fortunately, a highly effective model already exists. The 1975 Asilomar conference led to the establishment of the NIH Recombinant DNA Advisory Committee (RAC) to provide guidance and oversight of research using recombinant DNA (see, for example, R. M. Atlas Science 298, 753-754; 2002).

Piecemeal regulation will harm the advancement of science and wreak havoc on national security. Already the US Office of Management and Budget is planning to draft rules on the publication of some federal research classified as "sensitive homeland security information", but these rules will apply only to US government laboratories. In testimony before the House of Representatives Science Committee, several university officials said the proposed new rules would not serve the best interests of science.

A broadly based committee modelled on the RAC should be established now under the aegis of the NIH to develop guidelines for biomedical research with possible bioterrorism applications. A comparable committee should be formed in the US Department of Agriculture to deal with agricultural research. An interagency coordinating committee would be needed for supervision on security concerns related to governmentsponsored research.

An agenda for an RAC-like committee has been laid out by questions raised since last year's anthrax attacks. First, should access to genome databases be restricted? Would such restrictions prevent bioterrorism? More than 60 microbial genomes have already been deposited in publicly available genome databases and work is actively progressing on more than
150 more, as well as on several parasitic, fungal, plant and insect genomes.

Second, should scientists and scientific publishers practise self-censorship? For example, we now know that live poliovirus can be synthesized and that mail-order companies will provide the building-blocks necessary to reconstruct the virus in a laboratory. What, if any, restrictions on the publication of scientific findings are legitimate?

Third, should the training of scientists in the United States, whether US citizens or foreign nationals, be restricted? New US legislation requires universities and laboratories dealing with "select agents" to be registered with the US Department of Health and Human Services or the US Department of Agriculture and requires individual scientists to submit to background checks by the Department of Justice. Will an increasingly perilous legal landscape discourage legitimate scientists from engaging in research on infectious diseases?

The RAC model has served us well in the past; we should use it as the starting point for addressing these key policy questions to achieve effective and optimal guidance for the future.

Joseph G. Perpich

JG Perpich, LLC, 7315 Wisconsin Avenue, Suite 500

East, Bethesda, Maryland 20814, USA

\section{Modelling a new angle on understanding cancer}

Sir - There was a striking absence of discussion of quantitative methods in the recent interesting theoretical debate on the timing and nature of mutations controlling acquisition of the metastatic phenotype in invasive cancers (R. Bernards and R. A. Weinberg Nature 418, 823; 2002 and Correspondence Nature 419, 559-560; 2002). Without the discipline imposed by mathematical rigour, the arguments have to rely on intuitive reasoning and small pieces of data singled out from the vast extant literature. The reader is unable to independently evaluate the hypotheses or understand them in the context of all available experimental information or of theoretical models that organize information in integrally related phenomena such as carcinogenesis and tumour invasion. Although this state of affairs is normal in some disciplines such as tumour research, it would be unthinkable in the physical sciences.

Medical investigators often eschew mathematical models as too "simplistic" for the dynamics governing processes such as those in tumour biology. Instead, the general underlying principles in complex biological processes are expected to become apparent, as if by magic, once sufficient data are accumulated. Not surprisingly, this passive approach has failed to yield a comprehensive theoretical framework of understanding carcinogenesis, tumour invasion and metastatic behaviour. Clinical therapy thus remains largely empirical, based more on trial and error than a comprehensive understanding of biological first principles.

In most other scientific disciplines, there is an active search for broad generalizations through hypotheses framed in mathematical models that can be examined for internal consistency and compatibility with extant data. Predictions from these models can be tested by experiment and revised when necessary. Surely the success of this integrative approach, combining quantitative theoretical methods and experiment in highly complex systems in physics (including biophysics), chemistry, engineering and biological disciplines, such as ecology or structural biology, should motivate its enthusiastic application to other nonlinear biological processes such as carcinogenesis and tumour invasion.

In the absence of consistent application of rigorous mathematical models, theoretical medicine will largely remain empirical, phenomenological and anecdotal, successful only in linear systems that can be defined by a single experiment or a few experiments. Until the quantitative methods of the physical sciences are applied, many difficult and clinically important problems in tumour biology, including the dynamics that give rise to the metastatic phenotype, will remain unresolved. Robert A. Gatenby ${ }^{\star}$, Philip Maini $\dagger$ * Departments of Radiology and Applied Mathematics, University of Arizona, 1501 Campbell Avenue, Tucson, Arizona 85724, USA $\dagger$ Centre for Mathematical Biology, Mathematical Institute, 24-29 St Giles', Oxford University, Oxford OX1 3LB, UK

\section{Deserted by our geographical sense}

Sir - Your News in Brief on the Atacama Large Millimeter Array in Chile (Nature 419,$870 ; 2002$ ) sets a new record for imprecision. Chile is a narrow ribbon, with an average width of just $180 \mathrm{~km}$ and an eastern frontier of about $6,000 \mathrm{~km}$ of virtually constant longitude. Hence, referring to the observatory as located: "in the Atacama desert in the east of the country" is definitely too indefinite for anyone wanting to find it.

Gustavo Arteca

Département de Chimie et Biochimie, Laurentian University, Sudbury, Ontario P3E 2C6, Canada 